\title{
Using the A/T/N Framework to Examine Driving in Preclinical Alzheimer's Disease
}

\author{
Catherine M. Roe ${ }^{1, *}$, Ganesh M. Babulal ${ }^{1}$, Sarah H. Stout ${ }^{1}$, David B. Carr ${ }^{2}$, \\ Monique M. Williams ${ }^{3}$, Tammie L. S. Benzinger ${ }^{4}$, Anne M. Fagan ${ }^{5}$, David M. Holtzman ${ }^{5}$, \\ Beau M. Ances ${ }^{1}$ and John C. Morris ${ }^{6}$
}

1 Knight Alzheimer Disease Research Center, Department of Neurology, Washington University School of Medicine, St. Louis, MO 63110, USA; babulalg@wustl.edu (G.M.B.); shstout@wustl.edu (S.H.S.); bances@wustl.edu (B.M.A.)

2 Department of Medicine, Washington University School of Medicine, St. Louis, MO 63110, USA; dcarr@wustl.edu

3 VITAS Healthcare, St. Louis, MO 63110, USA; Monique.Williams@vitas.com

4 Knight Alzheimer Disease Research Center, Departments of Radiology and Neurosurgery, Washington University School of Medicine, St. Louis, MO 63110, USA; benzingert@wustl.edu

5 Knight Alzheimer Disease Research Center, Department of Neurology, the Hope Center for Neurological Disorders, Washington University School of Medicine, St. Louis, MO 63110, USA; fagana@wustl.edu (A.M.F.); holtzman@wustl.edu (D.M.H.)

6 Knight Alzheimer Disease Research Center, Departments of Neurology, Neurosurgery, Occupational Therapy, Pathology and Immunology, Physical Therapy, Washington University School of Medicine, St. Louis, MO 63110, USA; jcmorris@wustl.edu

* Correspondence: cathyr@wustl.edu; Tel.: +1-314-922-6470

Received: 11 March 2018; Accepted: 25 April 2018; Published: 2 May 2018

\begin{abstract}
The A/T/N classification system is the foundation of the 2018 NIA-AA Research Framework and is intended to guide the Alzheimer disease (AD) research agenda for the next 5-10 years. Driving is a widespread functional activity that may be particularly useful in investigation of functional changes in pathological $\mathrm{AD}$ before onset of cognitive symptoms. We examined driving in preclinical $\mathrm{AD}$ using the $\mathrm{A} / \mathrm{T} / \mathrm{N}$ framework and found that the onset of driving difficulties is most associated with abnormality of both amyloid and tau pathology, rather than amyloid alone. These results have implications for participant selection into clinical trials and for the application time of interventions aimed at prolonging the time of safe driving among older adults with preclinical AD.
\end{abstract}

Keywords: Alzheimer disease; preclinical Alzheimer disease; dementia; driving; biomarkers; cerebrospinal fluid; imaging

\section{Introduction}

By 2050, the United States will face a significant increase in the older adult population (age $>65$ years), a higher prevalence of Alzheimer disease (AD) and a related increase in older drivers (1 in 4 drivers) [1,2]. While age-related changes (e.g., cataracts, reduced reaction time) is a risk factor for driving decline, AD is also an important risk factor for higher crash risk and resulting injury and mortality [3-5]. Preclinical AD is defined as the long asymptomatic stage without cognitive decline but presents with biomarker evidence indicating that the pathophysiological process has begun [6]. Prior work has shown that cognitively normal older adults with preclinical AD make more errors and are faster to receive a marginal or fail rating on a standardized road test compared to those without preclinical $\mathrm{AD}[7,8]$. While past research has used a dichotomous classification of biomarker status (higher vs. lower) coupled with cognitive status, this study employed the A/T/N classification system 
to examine the relationship between $\mathrm{AD}$ biomarkers and driving performance as evaluated on a standardized road test $[7,8]$.

The A/T/N classification system [9] is the foundation of the new 2018 NIA-AA Research Framework [10], which was developed to guide observational and interventional research in AD. Participants are assigned to one of eight $\mathrm{A} / \mathrm{T} / \mathrm{N}$ profiles based on whether amyloid (A), tau (T) and neurodegeneration $(\mathrm{N})$ biomarkers are normal or abnormal. In turn, these eight profiles comprise four categories: AD (amyloid and tau both abnormal), AD pathophysiology (amyloid but not tau, abnormal) and normal (amyloid, tau and neurodegeneration normal) [10]. The framework is intended to guide the AD research agenda for the next 5-10 years [10].

Clinical trials testing potential treatments in the preclinical and early AD stages will be an important part of that agenda. These trials require evidence of both clinically meaningful cognitive and functional outcomes to demonstrate effectiveness.

Driving is a widespread functional activity that declines with symptomatic AD [8]. Recent work links driving and $\mathrm{AD}$ biomarker abnormalities among older adults who are cognitively normal $[8,11]$. Since the $\mathrm{A} / \mathrm{T} / \mathrm{N}$ framework is based solely on biomarkers, it may be particularly useful in investigation of functional changes in pathological AD before onset of cognitive symptoms. Thus, the purpose of this study is to examine driving in preclinical $\mathrm{AD}$ using the $\mathrm{A} / \mathrm{T} / \mathrm{N}$ framework.

\section{Materials and Methods}

Detailed information on study participants and methodology have been published [8]. Briefly, participants had normal cognition (Clinical Dementia Rating [CDR] [12] =0), were aged 65 years and older, drove an automobile at least once per week and had amyloid imaging and/or cerebrospinal fluid (CSF) collection within 2 years prior to, or 6 months after, the baseline assessment. At baseline and subsequent annual visits participants took part in clinical assessments, self-reported their current driving practices using the Driving Habits Questionnaire (DHQ) [13] and completed an on-road driving test. The Washington University Road Test (WURT) is a 45-min, in-traffic, predetermined route progressing from a quiet parking lot to more complex traffic conditions as safety permits [14]. The participant drives a mid-size sedan (with dual brakes), while the driving evaluator in the front seat provides directions, maintains vehicle safety and evaluates driving performance. At the end of the WURT, the participant receives a global rating (pass, marginal, fail) via a standardized scoring system. A pass rating is defined as minimal to no errors/no safety concerns where the driver demonstrates competency in all aspects of the test and across the different levels of traffic densities. A marginal rating is defined as moderate to minimal errors and some safety concerns where behaviors like rolling stops, inconsistent scanning or inability to maintain appropriate speed are observed but infrequent. A fail rating is defined as high to moderate errors and significant safety concerns where the driver demonstrates behaviors like failing to yield to a vehicle/pedestrian or running traffic light, which may lead to a crash anytime during the test. Since all participants were cognitively normal, a rating of fail is extremely rare, while a marginal rating may reveal some problems. As a result, the overall rating was dichotomized into marginal/fail and pass in the main analyses.

Ethical Statement. Study protocols were approved by the Washington University Human Research Protection Office (\#201706043) St. Louis, MO, USA and written informed consent was obtained. Investigations were carried out following the rules of the Declaration of Helsinki.

Statistical analyses. Each participant was assigned to one of the eight possible $\mathrm{A} / \mathrm{T} / \mathrm{N}$ profiles $(\mathrm{A}+/ \mathrm{T}+/ \mathrm{N}+, \mathrm{A}+/ \mathrm{T}+/ \mathrm{N}-, \mathrm{A}+/ \mathrm{T}-/ \mathrm{N}+, \mathrm{A}+/ \mathrm{T}-/ \mathrm{N}-, \mathrm{A}-/ \mathrm{T}+/ \mathrm{N}+, \mathrm{A}-/ \mathrm{T}+/ \mathrm{N}-, \mathrm{A}-/ \mathrm{T}-/ \mathrm{N}+$, $\mathrm{A}-/ \mathrm{T}-/ \mathrm{N}-)$ [10]. Amyloid abnormality on PET imaging was operationalized as a florbetapir (F-AV-45) standardized uptake value ratio (SUVR) of $\geq 1.219$ [11] or an SUVR $\geq 1.31$ for Pittsburgh Compound B [8]. An "A+" designation was assigned when either amyloid imaging or CSF A $\beta_{42}$ values (based on date and assay [8]) were abnormal. "T+" was assigned when CSF ptau $181 \geq 67 \mathrm{pg} / \mathrm{mL}$ and "N+" when tau $\geq 339 \mathrm{pg} / \mathrm{mL}$ [15]. Three A/T/N groups reflecting normal biomarkers, AD pathology, $\mathrm{AD}$ and suspected non-Alzheimer's pathology [SNAP] were then constructed based on the A/T/N 
classifications [10]. Age-adjusted differences between the A/T/N groups at baseline on everyday self-reported driving practices (driving space, miles driven, number of places visited and number of trips over the previous year) [13] were examined using general linear models. Kaplan-Meier curves and Cox proportional hazards models adjusting for age, tested differences in the time from baseline to first rating of Marginal or Fail (vs. Pass) on the yearly driving test as a function of A/T/N group. Linear mixed models, adjusted for age, tested self-reported decline over time on everyday driving practices assessed using the DHQ.

Given the current debate regarding whether SNAP is part of the AD pathological process, or reflects underlying tauopathy or other pathologies [16,17], we first analyzed the data excluding participants with SNAP, then reanalyzed the data including the SNAP group.

\section{Results}

A total of 157 participants with a mean \pm SD age of $72.6 \pm 4.9$ years and education of $16.3 \pm 2.6$ years met inclusion criteria (Table 1$)$. Seventy-three $(46.5 \%)$ were normal $(\mathrm{A}-/ \mathrm{T}-/ \mathrm{N}-)$, $25(15.9 \%)$ had AD pathology (A+), $25(15.9 \%)$ had AD (A+/T+) and $34(21.7 \%)$ had suspected non-Alzheimer's pathophysiology (SNAP [10]). Participants with longitudinal data $(\mathrm{N}=126)$ were followed up to 4.9 years (mean $\pm \mathrm{SD}=2.7 \pm 1.0$ ). Only two participants with marginal/fail ratings received a rating of fail (both within the first two years of follow-up), with the remaining participants who received marginal/fail ratings rated as marginal.

Table 1. Baseline demographic, biomarker and psychometric test performance for each group.

\begin{tabular}{|c|c|c|c|c|c|c|c|c|c|}
\hline & \multicolumn{2}{|c|}{$\operatorname{AD}(N=25)$} & \multicolumn{2}{|c|}{$\begin{array}{l}\text { AD Pathology } \\
\quad(\mathrm{N}=25)\end{array}$} & \multicolumn{2}{|c|}{ Normal $(\mathrm{N}=73)$} & \multicolumn{2}{|c|}{ SNAP $(N=34)$} & \multirow{2}{*}{$\begin{array}{c}\begin{array}{c}\text { Total } \\
(\mathrm{N}=157)\end{array} \\
p \text {-Value }\end{array}$} \\
\hline & Mean/N & $\mathrm{SD} / \%$ & Mean/N & $\mathrm{SD} / \%$ & Mean/N & $\mathrm{SD} / \%$ & Mean/N & $\mathrm{SD} / \%$ & \\
\hline \multicolumn{10}{|l|}{ Demographics } \\
\hline Age, y & 73.9 & 5.2 & 72.5 & 5.2 & 71.8 & 4.0 & 73.5 & 5.8 & 0.159 \\
\hline Education, $\mathrm{y}$ & 16.0 & 2.5 & 16.4 & 2.8 & 16.6 & 2.6 & 16.0 & 2.5 & 0.612 \\
\hline Women, $\mathrm{N}$ & 10 & $40.0 \%$ & 14 & $56.0 \%$ & 35 & $48.0 \%$ & 20 & $58.8 \%$ & 0.469 \\
\hline Race, $\mathrm{N}$ & & & & & & & & & 0.940 \\
\hline $\mathrm{AA}$ & 2 & $8.0 \%$ & 3 & $12.0 \%$ & 10 & $13.7 \%$ & 4 & $11.8 \%$ & \\
\hline Caucasian & 23 & $92.0 \%$ & 22 & $88.0 \%$ & 62 & $84.9 \%$ & 30 & $88.2 \%$ & \\
\hline More than one race & 0 & $0.0 \%$ & 0 & $0.0 \%$ & 1 & $1.4 \%$ & 0 & $0.0 \%$ & \\
\hline APOE4 & 13 & $52.0 \%$ & 12 & $48.0 \%$ & 13 & $17.8 \%$ & 34 & $17.7 \%$ & $<0.001$ \\
\hline \multicolumn{10}{|l|}{ Biomarker values } \\
\hline \multicolumn{10}{|l|}{ Amyloid positive, $\mathrm{N}$} \\
\hline Adjusted $\mathrm{A} \beta_{42}$ & -1.1 & 0.4 & -1.0 & 0.5 & 0.4 & 0.8 & 0.7 & 0.8 & $<0.001$ \\
\hline $\mathrm{tau}, \mathrm{pg} / \mathrm{mL}$ & 691.2 & 235.5 & 242.1 & 69.4 & 234.5 & 55.1 & 432.3 & 97.4 & $<0.001$ \\
\hline $\operatorname{ptau}_{181}, \mathrm{pg} / \mathrm{mL}$ & 111.2 & 30.7 & 43.7 & 11.1 & 46.3 & 9.8 & 77.5 & 20.2 & $<0.001$ \\
\hline \multicolumn{10}{|c|}{ Psychometric performance } \\
\hline SRT Free Recall & 29.7 & 8.2 & 32.3 & 4.8 & 32.4 & 5.6 & 31.9 & 5.5 & 0.249 \\
\hline Trailmaking A & 30.9 & 10.7 & 29.2 & 9.5 & 28.9 & 7.9 & 29.8 & 7.8 & 0.776 \\
\hline Trailmaking B & 83.0 & 33.3 & 75.7 & 29.3 & 76.1 & 30.5 & 79.5 & 30.8 & 0.805 \\
\hline Animal Naming & 20.3 & 6.9 & 20.3 & 4.9 & 22.0 & 6.0 & 21.4 & 5.1 & 0.630 \\
\hline
\end{tabular}

Abbreviations: $\mathrm{AD}=$ Alzheimer's disease; SNAP = Suspected non-Alzheimer Disease Pathophysiology; $\mathrm{SD}=$ standard deviation; $\mathrm{AA}=$ African American; SRT = Selective Reminding Test.

Analyses without the SNAP group. There were no differences between the A/T/N groups in everyday driving behaviors at baseline driving evaluation as reported on the DHQ $(p>0.212)$, nor in decline of DHQ-reported behaviors with time $(p>0.533)$. There were significant unadjusted (Log-rank test, $p=0.024$; Wilcoxon, $p=0.001$; Figure 1$)$ and adjusted $(p=0.031)$ effects of $\mathrm{A} / \mathrm{T} / \mathrm{N}$ group on time from baseline to a marginal/fail rating on the driving test. Participants with $\mathrm{AD}$ received a marginal/fail rating earlier than those with normal A/T / N biomarkers $(\mathrm{HR}=3.36,95 \% \mathrm{CI}=1.37-8.25$, $p=0.008)$, whereas there was no difference between those with normal $\mathrm{A} / \mathrm{T} / \mathrm{N}$ biomarkers and $\mathrm{AD}$ pathology $(\mathrm{HR}=1.53,95 \% \mathrm{CI}=0.59-4.02, p=0.385)$ in time to a marginal $/$ fail rating.

Analyses including the SNAP group. As in the previous analyses, the A/T/N groups did not differ in everyday driving behaviors at baseline driving evaluation $(p>0.108)$, nor in decline of these behaviors with time $(p>0.184)$. With inclusion of the SNAP group, the unadjusted (Log-rank test, 
$p=0.054$; Wilcoxon test $=0.004$; Figure 2$)$ and adjusted $(p=0.068) p$-values reflecting differences between the $\mathrm{A} / \mathrm{T} / \mathrm{N}$ groups on time from baseline to a marginal/fail rating on the driving test increased. Participants with AD received a marginal/fail rating earlier than those with normal A/T/N biomarkers ( $\mathrm{HR}=3.36,95 \% \mathrm{CI}=1.37-8.20, p=0.008)$, whereas there was no difference between those with normal A/T $/ \mathrm{N}$ biomarkers and AD pathology $(\mathrm{HR}=1.50,95 \% \mathrm{CI}=0.57-3.93, p=0.410)$ or SNAP $(\mathrm{HR}=1.42,95 \% \mathrm{CI}=0.60-3.35, p=0.429)$ in time to a marginal $/$ fail rating.

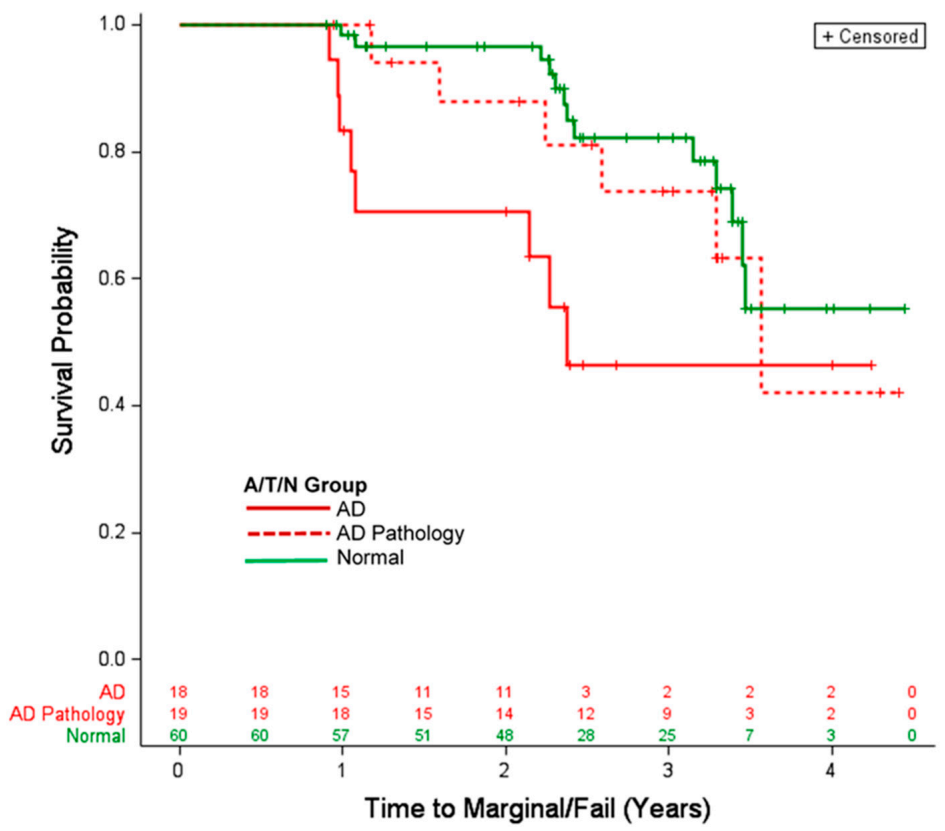

Figure 1. Time from baseline in years to receiving a Marginal or Fail rating as a function of $A / T / N$ parent group membership. Numbers below the $\mathrm{X}$-axis indicate the number of participants at risk at that time.

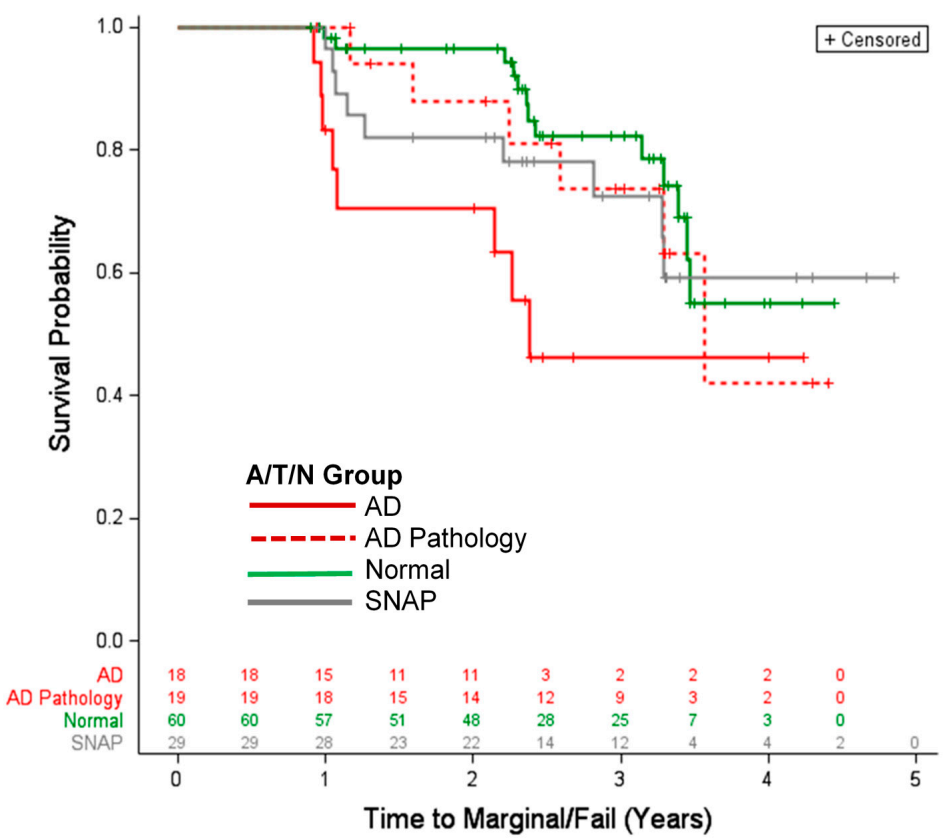

Figure 2. Time from baseline in years to receiving a Marginal or Fail rating as a function of $A / T / N$ parent group membership, including participants with suspected non-Alzheimer's pathophysiology (SNAP). Numbers below the $\mathrm{X}$-axis indicate the number of participants at risk at that time. 


\section{Discussion}

Examination of driving behavior using the $\mathrm{A} / \mathrm{T} / \mathrm{N}$ framework showed no associations between preclinical AD and cross-sectional, self-reported driving behavior at baseline, nor in changes in self-reported driving behavior with time. However, this framework predicted time to onset of objectively-measured driving problems on a standardized road test when only AD, AD pathology and normal groups were considered. Specifically, we found that the onset of driving difficulties is most associated with abnormality of both amyloid and tau pathology, rather than amyloid alone. These results suggest that in addition to cognition, the biomarker-based $\mathrm{A} / \mathrm{T} / \mathrm{N}$ framework may prove useful in guiding research that involves functional outcomes, such as driving.

Twenty-two percent of our participants fit into the SNAP group at baseline, a figure similar to that shown in other research samples of cognitively normal individuals [16,17]. There is uncertainty and controversy regarding what the SNAP group represents, with some arguing that given the relatively high prevalence of SNAP among cognitively normal older adults, it should be considered as part of the AD pathological spectrum and that the amyloid-centric view of AD is flawed. Others believe that SNAP may reflect distinct pathologies separate from AD, such as cerebrovascular disease, hippocampal sclerosis, or Parkinson's disease [16,17]. For this reason, we analyzed the data both with and without the SNAP group.

Upon reanalysis of the data including the SNAP group, the age-adjusted relationship between $\mathrm{A} / \mathrm{T} / \mathrm{N}$ and onset of driving problems was no longer significant. This is likely due to the relatively larger number of SNAP participants compared to the AD and AD pathology groups and the position of the SNAP survival curve relative to the others. As shown in Figure 2, the onset of driving difficulties among the SNAP groups driving behavior falls somewhere between that of the normal and AD groups. Using the onset of cognitive problems as an outcome, others have reported that the SNAP group tends to develop cognitive symptoms earlier than the persons in the normal group but later than those with biological AD [16,17].

As noted, most persons in our study who showed difficulty on the driving test received a "marginal" rating and the membership in the AD group should not be used as an indication that an individual should stop driving. Driving cessation is associated with several negative consequences, including higher rates of admission to long-term care facilities, higher prevalence of depression, faster decline in overall health and higher overall rates of mortality [18].

There are some limitations to this study. Importantly, the sample size of some of the A/T/N groups were small, with less than 20 participants with longitudinal data in each of the AD and AD pathology groups and therefore these results should be considered as preliminary. We will continue to follow this cohort to examine associations between the A/T/N framework and driving behavior among more people over a longer follow-up period. As with other standardized road tests [19,20], the WURT is a controlled condition with limited inference to actual daily driving behaviors and may be influenced by factors such as anxiety and rater subjectivity. Participants were predominately Caucasian, well-educated and willing to complete, biomarker testing for cerebrospinal fluid and amyloid imaging, annual clinical and neuropsychological testing and therefore they may not be representative of the general population. Since, the A/T/N framework is a newer AD classification system, further work is needed to confirm these findings on the relationship between driving and preclinical AD in larger and more diverse groups.

\section{Conclusions}

If replicated, these results may have implications for participant selection into clinical trials and for the application time of interventions aimed at prolonging the time of safe driving among older adults with preclinical AD. Because the onset of driving difficulties may be most associated with abnormality of both amyloid and tau pathology, rather than amyloid alone, our results suggest that the best time to introduce interventions may be once amyloid biomarkers are abnormal but before abnormalities are present in both tau and neurodegeneration. 
Author Contributions: C.M.R., G.M.B. and B.M.A. conceived the study; C.M.R., S.H.S., T.L.S.B., A.M.F., D.M.H. and J.C.M. contributed to acquisition of the data; C.M.R. analyzed the data; C.M.R. wrote the paper; D.B.C., M.M.W. and all coauthors contributed to providing important intellectual content and to revising the manuscript.

Funding: This research was funded by the National Institute on Aging [NIA, R01AG043434, R01AG43434-03S1, R01AG056466, P50AG005681, P01AG003991, P01AG026276]; Fred Simmons and Olga Mohan, the Farrell Family Research Fund and the Charles and Joanne Knight Alzheimer's Research Initiative of the Washington University Knight Alzheimer Disease Research Center (ADRC).

Acknowledgments: The authors thank the participants, investigators and staff of the Knight ADRC Clinical Core for participant assessments, Genetics Core for APOE genotyping, Biomarker Core for cerebrospinal fluid collection and analysis and the Imaging Core for amyloid imaging. Imaging facilities were supported by the Washington University Institute of Clinical and Translational Sciences grant UL1TR000448 from the National Center for Advancing Translational Sciences (NCATS) of the National Institutes of Health (NIH). Imaging analyses used the services of the Neuroimaging Informatics and Analysis Center, supported by NIH grant 5P30NS048056.

Conflicts of Interest: The authors declare no conflict of interest.

\section{References}

1. Alzheimer's Association. 2017 Alzheimer's disease facts and figures. Alzheimer's Dement. 2017, 13, 325-373.

2. Mizenko, A.J.; Tefft, B.C.; Arnold, L.S.; Grabowski, J. Older American Drivers and Traffic Safety Culture: A Longroad Study; AAA Foundation for Traffic Safety: Washington, DC, USA, 2014.

3. Cox, D.J.; Quillian, W.C.; Thorndike, F.P.; Kovatchev, B.P.; Hanna, G. Evaluating driving performance of outpatients with Alzheimer disease. J. Am. Board Fam. Pract. 1998, 11, 264-271. [CrossRef] [PubMed]

4. Duchek, J.M.; Hunt, L.; Ball, K.; Buckles, V.; Morris, J.C. Attention and driving performance in Alzheimer's disease. J. Gerontol. 1998, 53B, P130-P141. [CrossRef]

5. Reinach, S.J.; Rizzo, M.; McGehee, D.V. Driving with Alzheimer disease: The anatomy of a crash. Alzheimer Dis. Assoc. Disord. 1997, 11, 21-27. [CrossRef] [PubMed]

6. Sperling, R.A.; Aisen, P.S.; Beckett, L.A.; Bennett, D.A.; Craft, S.; Fagan, A.M.; Iwatsubo, T.; Jack, C.R., Jr.; Kaye, J.; Montine, T.J.; et al. Toward defining the preclinical stages of Alzheimer's disease: Recommendations from the national institute on aging-Alzheimer's association workgroups on diagnostic guidelines for Alzheimer's disease. Alzheimer's Dement. 2011, 7, 280-292. [CrossRef] [PubMed]

7. Roe, C.M.; Barco, P.P.; Head, D.M.; Ghoshal, N.; Selsor, N.; Babulal, G.M.; Fierberg, R.; Vernon, E.K.; Shulman, N.; Johnson, A.; et al. Amyloid imaging, cerebrospinal fluid biomarkers predict driving performance among cognitively normal individuals. Alzheimer Dis. Assoc. Disord. 2017, 31, 69-72. [CrossRef] [PubMed]

8. Roe, C.M.; Babulal, G.M.; Head, D.M.; Stout, S.H.; Vernon, E.K.; Ghoshal, N.; Garland, B.; Barco, P.P.; Williams, M.M.; Johnson, A.; et al. Preclinical Alzheimer's disease and longitudinal driving decline. Alzheimer's Dement. 2017, 3, 74-82. [CrossRef] [PubMed]

9. Jack, C.R.; Bennett, D.A.; Blennow, K.; Carrillo, M.C.; Feldman, H.H.; Frisoni, G.B.; Hampel, H.; Jagust, W.J.; Johnson, K.A.; Knopman, D.S.; et al. A/T/N: An unbiased descriptive classification scheme for Alzheimer disease biomarkers. Neurology 2016, 87, 539-547. [CrossRef] [PubMed]

10. Jack, C.R., Jr.; Bennett, D.A.; Blennow, K.; Carrillo, M.C.; Dunn, B.; Haeberlein, S.B.; Holtzman, D.M.; Jagust, W.; Jessen, F.; Karlawish, J.; et al. NIA-AA research framework: Toward a biological definition of Alzheimer's disease. Alzheimers Dement. 2018, 14, 535-562. [CrossRef] [PubMed]

11. Roe, C.M.; Babulal, G.M.; Mishra, S.; Gordon, B.A.; Stout, S.H.; Ott, B.R.; Carr, D.B.; Ances, B.M.; Morris, J.C.; Benzinger, T.L.S. Tau and amyloid positron emission tomography imaging predict driving performance among older adults with and without preclinical Alzheimer disease. J. Alzheimer's Dis. 2017, 3, 1-6.

12. Morris, J.C. The Clinical Dementia Rating (CDR): Current version and scoring rules. Neurology 1993, 43, 2412-2414. [CrossRef] [PubMed]

13. Owsley, C.; Stalvey, B.; Wells, J.; Sloane, M.E. Older drivers and cataract: Driving habits and crash risk. J. Gerontol. Ser. A Biol. Sci. Med. Sci. 1999, 54, M203-M211. [CrossRef]

14. Hunt, L.A.; Carr, D.; Duchek, J.M.; Grant, E.A.; Buckles, V.; Murphy, C.F.; Morris, J.C. Reliability and validity of the washington university road test: A performance-based assessment for drivers with dementia of the Alzheimer type. Arch. Neurol. 1997, 54, 707-712. [CrossRef] [PubMed] 
15. Vos, S.J.B.; Xiong, C.; Visser, P.J.; Jasielec, M.S.; Hassenstab, J.; Grant, E.A.; Cairns, N.J.; Morris, J.C.; Holtzman, D.M.; Fagan, A.M. Preclinical Alzheimer's disease and its outcome: A longitudinal cohort study. Lancet Neurol. 2013, 12, 957-965. [CrossRef]

16. Jack, C.R.; Knopman, D.S.; Chételat, G.; Dickson, D.; Fagan, A.M.; Frisoni, G.B.; Jagust, W.; Mormino, E.C.; Petersen, R.C.; Sperling, R.A.; et al. Suspected non-Alzheimer disease pathophysiology-concept and controversy. Nat. Rev. Neurol. 2016, 12, 117-124. [CrossRef] [PubMed]

17. Dani, M.; Brooks, D.J.; Edison, P. Suspected non Alzheimer's pathology-Is it non-Alzheimer's or non-amyloid? Ageing Res. Rev. 2017, 36, 20-31. [CrossRef] [PubMed]

18. Liddle, J.; Bennett, S.; Allen, S.; Lie, D.C.; Standen, B.; Pachana, N.A. The stages of driving cessation for people with dementia: Needs and challenges. Int. Psychogeriatr. 2013, 25, 2033-2046. [CrossRef] [PubMed]

19. Eby, D.W.; Silverstein, N.M.; Molnar, L.J.; LeBlanc, D.; Adler, G. Driving behaviors in early stage dementia: A study using in-vehicle technology. Accid. Anal. Prev. 2012, 49, 330-337. [CrossRef] [PubMed]

20. Hird, M.A.; Egeto, P.; Fischer, C.E.; Naglie, G.; Schweizer, T.A.; Pachana, N. A systematic review and meta-analysis of on-road simulator and cognitive driving assessment in Alzheimer's disease and mild cognitive impairment. J. Alzheimer's Dis. 2016, 53, 713-729. [CrossRef] [PubMed]

(C) 2018 by the authors. Licensee MDPI, Basel, Switzerland. This article is an open access article distributed under the terms and conditions of the Creative Commons Attribution (CC BY) license (http://creativecommons.org/licenses/by/4.0/). 